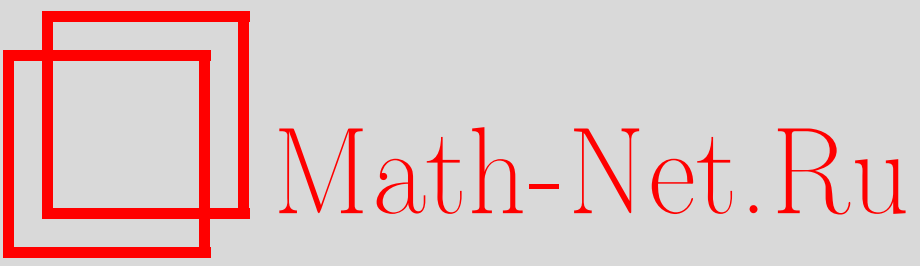

И. А. Соловьев, Уравнение для волн вероятности, моделирующих поведение случайных величин, средние значения которых описываются системой обыкновенных дифференциальных уравнений первого порядка, ТМФ, 1997, том 111, номер 3, 356-368

DOI: https://doi.org/10.4213/tmf1014

Использование Общероссийского математического портала Math-Net.Ru подразумевает, что вы прочитали и согласны с пользовательским соглашением

http: //www.mathnet.ru/rus/agreement

Параметры загрузки:

IP : 3.93 .64 .190

26 апреля 2023 г., 18:05:53 


\section{УРАВНЕНИЕ ДЛЯ ВОЛН ВЕРОЯТНОСТИ, МОДЕЛИРУЮЩИХ ПОВЕДЕНИЕ СЛУЧАЙНЫХ ВЕЛИЧИН, СРЕДНИЕ ЗНАЧЕНИЯ КОТОРЫХ ОПИСЫВАЮТСЯ СИСТЕМОЙ ОБЫКНОВЕННЫХ ДИФФЕРЕНЦИАЛЬНЫХ УРАВНЕНИЙ ПЕРВОГО ПОРЯДКА}

Изложена теория волн вероятностей применительно к исследованию поведения стохастических векторных величин, средние значения компонент которых подчиняются обыкновенным дифференциальным уравнениям первого порядка. Приведено уравнение для волн вероятности, соответствующее дифференциальной модели для средних величин. Рассмотрена связь этого уравнения с уравнением Лиувилля. Доказан аналог теоремы Эренфеста. Получено дифференциальное уравнение первого порядка для изменения дисперсии компоненты случайной векторной величины. Обсуждается проблема интерпретации постоянной Планка. Определены условия, при которых распространение волновых пакетов происходит с нарастающей или убывающей дисперсией.

\section{ВВЕДЕНИЕ}

Дифференциальные уравнения для стохастических величин привлекли внимание после появления известных работ [1], посвященных броуновскому движению. Простейшим случаем таких уравнений является уравнение Ланжевена [2], которое может быть представлено в виде

$$
\frac{1}{2} m \frac{d^{2}\left\langle x^{2}\right\rangle}{d t^{2}}-m\left(\left\langle\frac{d x}{d t}\right\rangle\right)^{2}+3 \pi \eta a \frac{d\left\langle x^{2}\right\rangle}{d t}=\langle x X\rangle
$$

где $\langle\ldots\rangle$ - среднее значение, $m$ - масса частищы, $a$ - ее диаметр, $\eta$ - вязкость жидкости, в которой находится частица, $X$ - флуктуационная сила, обусловленная толчками молекул жидкости, $x$ - положение частицы, $t$ - время.

В последние годы многие ставшие уже классическими дифференциальные модели физики, химии, биологии, медицины, экологии, социологии, экономики, использовавшиеся ранее для получения детерминированных решений, начинают преобразовываться в

* Московский энергетический институт (технический университет), Москва, Россия 
стохастические. Библиография по таким трансформациям постановок задач представлена в [3]. Среди них отметим систему уравнений Вольтерра-Лотки, первоначально предназначенную для описания в детерминированном приближении широкого класса явлений, моделирующих процессы рождения и гибели в терминах численности “жертв" $\langle x\rangle$ и “хищников" $\langle y\rangle$ :

$$
\begin{aligned}
& \frac{d\langle x\rangle}{d t}=a\langle x\rangle-\alpha\langle x\rangle\langle y\rangle, \\
& \frac{d\langle y\rangle}{d t}=\alpha\langle x\rangle\langle y\rangle-b\langle y\rangle .
\end{aligned}
$$

В реальности величины численностей “жертв" и “хишников" флуктуируют около своих средних значений $\langle x\rangle$ и $\langle y\rangle$. Учет флуктуаций можно произвести, например, с помощью распределения вероятностей $P(x, y, t)$ численности особей в данный момент времени $t$, удовлетворяюшего уравнению

$$
\begin{aligned}
\frac{\partial P}{\partial t}= & a(x-1) P(x-1, y, t)+\alpha(x+1)(y-1) P(x+1, y-1, t)+ \\
& +b(y+1) P(x . y+1, t)-(a x+\alpha x y+b y) P(x, y, t) .
\end{aligned}
$$

Отметим, что это не единственный способ учета стохастичности процесса взаимодействия популяций. В общей теории исследования случайных величин абстрактной природы, чьи усредненные значения подчиняются дифференциальным уравнениям, можно выделить три основных взаимно дополняюших направления.

Первое из направлений представлено в трудах Фоккера [4], Планка [5], Колмогорова [6] и других. В этих работах построены общие уравнения для плотностей вероятностей случайных величин, математические ожидания которых описываются дифференциальными уравнениями.

Начало второму направлению - построению стохастических интегралов для стохастических дифференциальных уравнений, положено в исследованиях Ито [7] и Стратоновича [8]. В ряде случаев методами Ито и Стратоновича характеристики случайных величин получаются проще, чем с помощью уравнений Фоккера-Планка.

Наконец, третье направление исследования случайных процессов появилось в ходе дискуссий об интерпретации квантовой механики [9]. Важную роль при вероятностном истолковании механики микромира сыграли теоремы Эренфеста [10].

Если первые два направления широко используются при моделировании случайных процессов, то область применимости третьего направления, на наш взгляд, необоснованно сужена до трактовки лишь явлений квантовой физики.

С одной стороны, это связано с тем, что уравнения Шредингера были построены только для специфических физических явлений. Для произвольных случайных величин с известными дифференциальными уравнениями относительно средних значений аналоги уравнения Шредингера получены не были. Особую трудность при этом представляют поиск и интерпретация постоянной, подобной постоянной Планка.

С другой стороны, в дискуссиях по поводу вероятностной трактовки волновой механики остается открытой проблема построения волн вероятности из “чисто” вероятностных соображений [9]. 
Настоящая работа посвящена исследованию стохастических векторных величин $\bar{q}(t)=\left(q_{1}(t), \ldots, q_{n}(t)\right)^{T}$, средние значения компонент которых подчиняются обыкновенным дифференциальным уравнениям первого порядка

$$
d\left\langle q_{j}\right\rangle / d t=\left\langle f_{j}\left(q_{1}, \ldots, q_{n}, t\right)\right\rangle, \quad j=1,2, \ldots, n .
$$

Условимся назьвать $q_{j} j$-й обобшенной координатой абстрактной материальной частицы, а $t$-обобшенным временем. Если реальные значения $t \in[a, \infty)$, то преобразованием $t^{\prime}=t-a$ приведем область изменения этой величины к $[0, \infty)$.

В отличие от публикаций $[11,12,13]$, в которых плотность вероятности находится из соответствуюших (1) уравнений Фоккера-Планка или методами Ито-Стратоновича, в настоящей работе развивается подход волн вероятности, предложенный в статьях $[14,15]$. Этот подход позволяет каждой обобщенной частище, среднее значение обобщенного перемешения которой $\langle\bar{q}(t)\rangle$ моделируется уравнениями $(1)$, поставить в соответствие в условном пространстве-времени $\Omega\left(q_{1}, q_{2}, \ldots, q_{n}, t\right)$ волновой пакет с частотой и волновым числом, отвечаюшими поведению среднего значения $\langle\bar{q}(t)\rangle$.

\section{1. ОПРЕДЕЛЕНИЕ ВОЛН ВЕРОЯТНОСТИ И ФОРМУЛИРОВКА УСЛОВИЙ ИХ СУЩЕСТВОВАНИЯ}

ОПРЕДЕЛЕНИЕ 1 . Волнами вероятности для случайного вектора $\bar{q}(t)$, компоненты которого удовлетворяют уравнениям (1), называются две комплексно-сопряженные функции $\Psi(\bar{q}, t)$ и $\Psi^{*}(\bar{q}, t)$, являюшиеся нетривиальными $\left(\Psi \neq \equiv 0, \Psi^{*} \not \equiv 0\right)$ решениями уравнений

$$
\begin{array}{r}
i \hbar_{q} \frac{\partial \Psi}{\partial t}+\sum_{k=1}^{n}\left(i \hbar_{q} f_{k}(\bar{q}, t) \frac{\partial \Psi}{\partial q_{k}}+\frac{1}{2} i \hbar_{q} \Psi \frac{\partial f_{k}}{\partial q_{k}}\right)-U(\bar{q}, t) \Psi=0 \\
i \hbar_{q} \frac{\partial \Psi^{*}}{\partial t}+\sum_{k=1}^{n}\left(i \hbar_{q} f_{k}(\bar{q}, t) \frac{\partial \Psi^{*}}{\partial q_{k}}+\frac{1}{2} i \hbar_{q} \Psi^{*} \frac{\partial f_{k}}{\partial q_{k}}\right)+U(\bar{q}, t) \Psi^{*}=0
\end{array}
$$

Здесь $t>0, \quad \bar{q} \in \mathbb{R}^{n}$, а $\hbar_{q}$ - некоторый параметр, равный кванту аналога действия, построенного с помошью $\bar{q}(t)$. Связь $\hbar_{q}$ с начальной дисперсией величины $\bar{q}(t)$ будет обсуждаться в разделе 3 . Функция $U(\bar{q}, t)$ - аналог потенциальной энергии, в данной постановке может быть выбрана произвольной среди функций, для которых существуют решения уравнений (2) и (3), отвечаюшие волновым характеристикам обобщенной частицы. В ряде случаев удобно считать, что $U(\bar{q}, t) \in C^{\infty}\left[\mathbb{R}^{n+1}\right]$, в частности $U(\bar{q}, t)=$ const.

ОПРЕДЕлЕНИЕ 2 . Произведение $\Psi(\bar{q}, t) \Psi^{*}(\bar{q}, t)$ называется плотностью вероятности и обозначается $P(\bar{q}, t)$.

Поскольку “частица", описываемая случайным вектором $\bar{q}(t)$, в каждый момент $t$ обязательно находится где-то в обобщенном пространстве $\mathbb{R}^{n}$, то вероятность ее обнаружения не должна зависеть от $t$ и обязана равняться единице. Таким образом, несобственный интеграл

$$
\int_{-\infty}^{\infty} \cdots \int_{-\infty}^{\infty} \Psi(\bar{q}, t) \Psi^{*}(\bar{q}, t) d q_{1} d q_{2} \ldots d q_{n}=1
$$


должен сходиться и не должен зависеть от обобщенного времени $t$. Достаточные условия этого сформулированы в теореме 1.

ТЕОрема 1. Если $\Psi$ и $\Psi^{*}$ - нетривиальные решения уравнений (2) и (3) и если для любых $t \geq 0$ и $j=1,2, \ldots, n$ выполняется равенство

$$
\lim _{i=1,2, \ldots, n} f_{j}(\bar{q}, t) \Psi(\bar{q}, t) \Psi^{*}(\bar{q}, t)=0
$$

в частности, если $\left|f_{j}(\bar{q}, t)\right| \leq M=$ const $n р и a \leq q \leq b(M>0) u f_{j}(\bar{q}, t) \equiv 0$ при $q<$ а и $q>b$, то интеграл в (4) сходится и не зависит от обобщенного времени $t$.

ДокАЗАТЕЛЬСтво. Умножим уравнения (2) и (3) соответственно на $\Psi^{*}$ и $\Psi$ и сложим полученные равенства. Имеем

$$
i \hbar_{q} \frac{\partial\left(\Psi \Psi^{*}\right)}{\partial t}+\sum_{j=1}^{n} i \hbar_{q} \frac{\partial\left(\Psi \Psi^{*} f_{j}(\bar{q}, t)\right)}{\partial q_{j}}=0
$$

Проинтегрировав обе части последнего соотношения по $q_{j}$ и воспользовавшись условием теоремы, получим следуюшую цепочку равенств:

$$
\begin{aligned}
& i \hbar_{q} \int_{-\infty}^{\infty} \ldots \int_{-\infty}^{\infty} \partial\left(\Psi \Psi^{*}\right) / \partial t d q_{1} \ldots d q_{n}+ \\
& \quad+i \hbar_{q} \sum_{j=1}^{n} \int_{-\infty}^{\infty} \ldots \int_{-\infty}^{\infty} \ldots \int_{-\infty}^{\infty} \partial\left(f_{j}(\bar{q}, t) \Psi \Psi^{*}\right) / \partial q_{j} d q_{1} \ldots d q_{j} \ldots d q_{n}=0 \\
& \quad i \hbar_{q} \int_{-\infty}^{\infty} \ldots \int_{-\infty}^{\infty} \partial\left(\Psi \Psi^{*}\right) / \partial t d q_{1} \ldots d q_{n}+ \\
& \quad+\left.i \hbar_{q} \sum_{j=1}^{n} \int_{-\infty}^{\infty} \ldots \int_{-\infty}^{\infty} \lim _{b_{j} \rightarrow \infty} f_{j}\left(q_{1}, \ldots, q_{j}, \ldots, q_{n}, t\right) \Psi \Psi^{*}\right|_{a_{j}} ^{b_{j}} d q_{1} \times \\
& \quad \times d q_{2} \ldots d q_{j-1} d q_{j+1} \ldots d q_{n}=0 \\
& \quad i \hbar_{q} \int_{-\infty}^{\infty} \ldots \int_{-\infty}^{\infty} \partial\left(\Psi \Psi^{*}\right) / \partial t d q_{1} \ldots d q_{n}=0 .
\end{aligned}
$$

В силу теоремы о равномерной сходимости несобственного интеграла, зависящего от параметра, заключаем, что для всех $t \geq 0$ интеграл сходится:

$$
J=\int_{-\infty}^{\infty} \cdots \int_{-\infty}^{\infty} \Psi \Psi^{*} d q_{1} \ldots d q_{n}
$$

Отметим, что $J \neq 0$, т.к. в противном случае $\Psi$ и $\Psi^{*}$ были бы тривиальными решениями уравнений $(2)$ и (3). Если $J \neq 0$, то, разделив $\Psi$ и $\Psi^{*}$ на $\sqrt{J}$, получим новые выражения для волн вероятности, которые удовлетворяют условию нормировки (4). 
Условие нормировки можно сформулировать и с помощью собственных интегралов в виде так называемой "нормировки в ящике":

$$
\int_{-L_{1} / 2}^{L_{1} / 2} \cdots \int_{-L_{n} / 2}^{L_{n} / 2} \Psi(\bar{q}, t) \Psi^{*}(\bar{q}, t) d q_{1} \ldots d q_{n}=1 .
$$

Для выполнения условий (4) и (5) потребуем, чтобы функции $\Psi$ и $\Psi^{*}$ искались среди таких решений уравнений $(2)$ и $(3)$, которые как функции от $\bar{q}$ принадлежали бы пространству $L_{2}: \Psi(\bar{q}, t) \in L_{2}\left[\mathbb{R}^{n}\right], \Psi^{*}(\bar{q}, t) \in L_{2}\left[\mathbb{R}^{n}\right]$. Функции $\Psi(\bar{q}, t)$ и $\Psi^{*}(\bar{q}, t)$ должны удовлетворять начальным условиям вида

$$
\Psi(\bar{q}, 0)=\varphi\left(\bar{q}, \hbar_{q}\right), \quad \Psi^{*}(\bar{q}, 0)=\varphi^{*}\left(\bar{q}, \hbar_{q}\right)
$$

где

$$
\int_{-\infty}^{\infty} \cdots \int_{-\infty}^{\infty} \varphi\left(\bar{q}, \hbar_{q}\right) \varphi^{*}\left(\bar{q}, \hbar_{q}\right) d q_{1} \ldots d q_{n}=1
$$

или

$$
\int_{-L_{1} / 2}^{L_{1} / 2} \cdots \int_{-L_{n} / 2}^{L_{n} / 2} \varphi\left(\bar{q}, \hbar_{q}\right) \varphi^{*}\left(\bar{q}, \hbar_{q}\right) d q_{1} \ldots d q_{n}=1 .
$$

Кроме того, $\Psi(\bar{q}, t)$ и $\Psi^{*}(\bar{q}, t)$ должны удовлетворять либо граничным условиям на бесконечности:

$$
\begin{array}{r}
\lim _{q_{j} \rightarrow \pm \infty} \Psi\left(q_{1}, \ldots, q_{j}, \ldots, q_{n}, t\right) q_{j}^{2} f_{j}\left(q_{1}, \ldots, q_{j}, \ldots, q_{n}, t\right)=0, \\
\lim _{q_{j} \rightarrow \pm \infty} \Psi^{*}\left(q_{1}, \ldots, q_{j}, \ldots, q_{n}, t\right) q_{j}^{2} f_{j}\left(q_{1}, \ldots, q_{j}, \ldots, q_{n}, t\right)=0,
\end{array}
$$

либо периодическим граничным условиям:

$$
\begin{aligned}
\Psi\left(q_{1}, \ldots, q_{j}\right. & \left.=-L_{j} / 2, \ldots, q_{n}, t\right)=\Psi\left(q_{1}, \ldots, q_{j}=L_{j} / 2, \ldots, q_{n}, t\right)=0, \\
\Psi^{*}\left(q_{1}, \ldots, q_{j}\right. & \left.=-L_{j} / 2, \ldots, q_{n}, t\right)=\Psi^{*}\left(q_{1}, \ldots, q_{j}=L_{j} / 2, \ldots, q_{n}, t\right)=0 .
\end{aligned}
$$

В процессе доказательства теоремы 1 получено уравнение Лиувилля. Таким образом, справедлива следуюшая теорема.

ТеОрема 2. Плотность вероятности $P(\bar{q}, t)=\Psi(\bar{q}, t) \Psi^{*}(\bar{q}, t)$, где функиии $\Psi(\bar{q}, t)$ $u \Psi^{*}(\bar{q}, t)$ подчиняются уравнениям (2) $и(3)$, удовлетворяет уравнению Лиувилля:

$$
\partial P / \partial t+\sum_{k=1}^{n} \partial\left(f_{k}(\bar{q}, t) P(\bar{q}, t)\right) / \partial q_{k}=0 .
$$




\section{2. ТЕОРЕМЫ О ПОВЕДЕНИИ СРЕДНИХ ЗНАЧЕНИЙ И ДИСПЕРСИИ}

ОПРЕДЕЛЕНИЕ 3 . Средним значением $\langle f(\bar{q}, t)\rangle$ скалярной функции, зависящей от компонент вектора $\bar{q}=\left(q_{1}, \ldots, q_{n}\right)$, называется

$$
\langle f(\bar{q}(t), t)\rangle=\int_{-\infty}^{\infty} \cdots \int_{-\infty}^{\infty} \Psi(\bar{q}, t) f(\bar{q}, t) \Psi^{*}(\bar{q}, t) d q_{1} \ldots d q_{n}
$$

В частности, среднее значение $\left\langle q_{i}(t)\right\rangle$ для компоненты $q_{i}(t)$ имеет вид

$$
\left\langle q_{k}(t)\right\rangle=\int_{-\infty}^{\infty} \cdots \int_{-\infty}^{\infty} \Psi(\bar{q}, t) q_{k} \Psi^{*}(\bar{q}, t) d q_{1} \ldots d q_{n}
$$

ОПРЕДЕлЕниЕ 4. Средними значениями операторов “обобщенной энергии” $i \hbar_{q} \partial / \partial t$ и “ $i$-й компоненты обобшенного импульса" $-i \hbar_{q} \partial / \partial q_{i}$ называются соответственно следуюшие величины:

$$
\begin{aligned}
\left\langle i \hbar_{q}(\partial / \partial t)\right\rangle & =\int_{-\infty}^{\infty} \cdots \int_{-\infty}^{\infty} \Psi^{*}(\bar{q}, t)\left(i \hbar_{q} \partial \Psi(\bar{q}, t) / \partial t\right) d q_{1} \ldots d q_{n} \\
\left\langle-i \hbar_{q}\left(\partial / \partial q_{k}\right)\right\rangle & =\int_{-\infty}^{\infty} \cdots \int_{-\infty}^{\infty} \Psi^{*}(\bar{q}, t)\left(-i \hbar_{q} \partial \Psi(\bar{q}, t) / \partial q_{k}\right) d q_{1} \ldots d q_{n} .
\end{aligned}
$$

ТЕОРема 3. Среднее значение $\left\langle q_{j}(t)\right\rangle$ j-й компоненты случайного вектора $\bar{q}(t)$, поведение которого моделируется с помощью нетривиальных решений уравнений (2) и (3) и условий (4), (6)-(9), подчиняется уравнению (1).

ДокАЗАТЕльСтво. Найдем производную по $t$ и от среднего значения $\left\langle q_{j}(t)\right\rangle$ :

$$
\begin{aligned}
\frac{d\left\langle q_{j}(t)\right\rangle}{d t}= & \frac{d}{d t}\left(\int_{-\infty}^{\infty} \cdots \int_{-\infty}^{\infty} \Psi(\bar{q}, t) q_{j} \Psi^{*}(\bar{q}, t) d q_{1} \ldots d q_{n}\right)= \\
= & \int_{-\infty}^{\infty} \cdots \int_{-\infty}^{\infty} \frac{d}{d t}\left(\Psi(\bar{q}, t) q_{j} \Psi^{*}(\bar{q}, t)\right) d q_{1} \ldots d q_{n}= \\
= & \int_{-\infty}^{\infty} \cdots \int_{-\infty}^{\infty} \frac{d}{d t}(\Psi(\bar{q}, t)) q_{j} \Psi^{*}(\bar{q}, t) d q_{1} \ldots d q_{n}+ \\
& +\int_{-\infty}^{\infty} \cdots \int_{-\infty}^{\infty} \Psi(\bar{q}, t) q_{j} \frac{d}{d t} \Psi^{*}(\bar{q}, t) d q_{1} \ldots d q_{n} .
\end{aligned}
$$


Учтя уравнения (2) и (3), получим

$$
\begin{aligned}
\frac{d\left\langle q_{j}(t)\right\rangle}{d t}=- & \int_{-\infty}^{\infty} \ldots \int_{-\infty}^{\infty}\left(\sum_{k=1}^{n} f_{k}(\bar{q}, t) \frac{\partial \Psi(\bar{q}, t)}{\partial q_{k}}+\right. \\
& \left.+\frac{1}{2} \Psi(\bar{q}, t) \frac{\partial f_{k}}{\partial q_{k}}+\left(i / \hbar_{q}\right) U \Psi(\bar{q}, t)\right) q_{j} \Psi^{*}(\bar{q}, t) d q_{1} \ldots d q_{n}- \\
& -\int_{-\infty}^{\infty} \cdots \int_{-\infty}^{\infty} \Psi(\bar{q}, t) q_{j}\left(\sum_{k=1}^{n} f_{k}(\bar{q}, t) \frac{\partial \Psi^{*}(\bar{q}, t)}{\partial q_{k}}+\frac{1}{2} \Psi^{*}(\bar{q}, t) \frac{\partial f_{k}}{\partial q_{k}}-\right. \\
& \left.-\left(i / \hbar_{q}\right) U \Psi^{*}(\bar{q}, t)\right) d q_{1} \ldots d q_{n}= \\
= & \int_{-\infty}^{\infty} \ldots \int_{-\infty}^{\infty}\left\{\sum_{k=1}^{n} f_{k} \frac{\partial \Psi^{*}(\bar{q}, t)}{\partial q_{k}} q_{j} \Psi(\bar{q}, t)+\right. \\
& \left.+\Psi^{*}(\bar{q}, t) q_{j} f_{k} \frac{\partial \Psi(\bar{q}, t)}{\partial q_{k}}+\Psi(\bar{q}, t) q_{j} \Psi^{*}(\bar{q}, t) \frac{\partial f_{k}}{\partial q_{k}}\right\} d q_{1} \ldots d q_{n}= \\
= & \int_{-\infty}^{\infty}\left\{\sum_{k=1}^{n} f_{k} q_{j} \frac{\partial\left(\Psi \Psi^{*}\right)}{\partial q_{k}}+\Psi q_{j} \Psi^{*} \frac{\partial f_{k}}{\partial q_{k}}\right\} d q_{1} \ldots d q_{n} .
\end{aligned}
$$

Первое слагаемое в фигурных скобках можно проинтегрировать по частям по $q_{k}$. С учетом (8) и (9) получим

$$
\begin{aligned}
\int_{-\infty}^{\infty} & \ldots \int_{-\infty}^{\infty} \sum_{k=1}^{n} q_{j} f_{k} \frac{\partial\left(\Psi \Psi^{*}\right)}{\partial q_{k}} d q_{1} \ldots d q_{n}= \\
= & \left.\int_{-\infty}^{\infty} \ldots \int_{-\infty}^{\infty} \lim _{\substack{b \rightarrow \infty \\
a \rightarrow-\infty}} \sum_{k=1}^{n}\left(\Psi q_{j} f_{k} \Psi^{*}\right)\right|_{q_{k}=a} ^{q_{k}=b} d q_{1} \ldots d q_{k-1} d q_{k+1} \ldots d q_{n}- \\
& -\int_{-\infty}^{\infty} \ldots \int_{-\infty}^{\infty} \sum_{k=1}^{n} \frac{\partial\left(q_{j} f_{k}\right)}{\partial q_{k}} \Psi^{*} \Psi d q_{1} \ldots d q_{n}= \\
= & -\int_{-\infty}^{\infty} \ldots \int_{-\infty}^{\infty} \sum_{k=1}^{n}\left\{\frac{\partial f_{k}}{\partial q_{k}} \Psi^{*} q_{j} \Psi+\Psi^{*} f_{j} \Psi\right\} d q_{1} \ldots d q_{n}
\end{aligned}
$$

Подставив это выражение в соотношение (10), придем к уравнениям (1).

ТЕОрема 4. Дисперсия $\sigma_{j}^{2}=\left\langle q_{j}^{2}(t)\right\rangle-\left\langle q_{j}(t)\right\rangle^{2}$ решения $j$-го уравнения системь (1) подчиняется следующему дифференииальному уравнению:

$$
d\left(\sigma_{j}^{2}\right) / d t=2\left(\left\langle f_{j}(\bar{q}, t) q_{j}\right\rangle-\left\langle q_{j}\right\rangle\left\langle f_{j}(\bar{q}, t)\right\rangle\right)
$$


ДокАЗАТЕЛЬСТво. Имеем

$$
d\left(\sigma_{j}^{2}\right) / d t=\frac{d}{d t}\left(\int_{-\infty}^{\infty} \cdots \int_{-\infty}^{\infty} \Psi^{*}(\bar{q}, t) q_{j}^{2} \Psi(\bar{q}, t) d q_{1} \ldots d q_{n}\right)-2\left\langle q_{j}(t)\right\rangle \frac{d}{d t}\left\langle q_{j}(t)\right\rangle .
$$

Подставим в (12) производную $d\left\langle q_{j}(t)\right\rangle / d t$ из (1) и внесем эту производную под знак интеграла в первом слагаемом. Тогда получится

$$
\begin{aligned}
d\left(\sigma_{j}^{2}\right) / d t= & \int_{-\infty}^{\infty} \cdots \int_{-\infty}^{\infty}\left(\partial \Psi^{*}(\bar{q}, t) / \partial t\right) q_{j}^{2} \Psi(\bar{q}, t) d q_{1} \ldots d q_{n}+ \\
& +\int_{-\infty}^{\infty} \cdots \int_{-\infty}^{\infty} \Psi^{*}(\bar{q}, t) q_{j}^{2}(\partial \Psi(\bar{q}, t) / \partial t) d q_{1} \ldots d q_{n}- \\
& -2\left\langle q_{j}(t)\right\rangle\left(d\left\langle q_{j}(t)\right\rangle / d t\right) .
\end{aligned}
$$

Учитывая опять уравнение (2) и (3), имеем

$$
\begin{aligned}
\int_{-\infty}^{\infty} & \ldots \int_{-\infty}^{\infty} \sum_{k=1}^{n}\left(-f_{k} \frac{\partial \Psi}{\partial q_{k}}-\frac{1}{2} \Psi \frac{\partial f_{k}}{\partial q_{k}}-\left(i / \hbar_{q}\right) U \Psi\right) q_{j}^{2} \Psi^{*} d q_{1} \ldots d q_{n}+ \\
& +\int_{-\infty}^{\infty} \cdots \int_{-\infty}^{\infty} \Psi q_{j}^{2}\left(\sum_{k=1}^{n}\left(-f_{k} \frac{\partial \Psi^{*}}{\partial q_{k}}-\frac{1}{2} \Psi^{*} \frac{\partial f_{k}}{\partial q_{k}}+\left(i / \hbar_{q}\right) U \Psi^{*}\right)\right) d q_{1} \ldots d q_{n}= \\
= & -\int_{-\infty}^{\infty} \cdots \int_{-\infty}^{\infty}\left\{\sum_{k=1}^{n} f_{k} \frac{\partial \Psi}{\partial q_{k}} q_{j}^{2} \Psi^{*}+\Psi q_{j}^{2} f_{k} \frac{\partial \Psi^{*}}{\partial q_{k}}+\Psi q_{j}^{2} \Psi^{*} \frac{\partial f_{k}}{\partial q_{k}}\right\} d q_{1} \ldots d q_{n}= \\
= & -\int_{-\infty}^{\infty} \sum_{k=1}^{n}\left\{f_{k} q_{j}^{2} \frac{\partial\left(\Psi \Psi^{*}\right)}{\partial q_{k}}+\Psi q_{j}^{2} \Psi^{*} \frac{\partial f_{k}}{\partial q_{k}}\right\} d q_{1} \ldots d q_{n} .
\end{aligned}
$$

Интегрируя по частям первое слагаемое в фигурных скобках (14), с учетом (8) и (9) получим

$$
\begin{aligned}
\int_{-\infty}^{\infty} & \ldots \int_{-\infty}^{\infty} q_{j}^{2} f_{k} \frac{\partial\left(\Psi \Psi^{*}\right)}{\partial q_{k}} d q_{1} \ldots d q_{n}= \\
= & \left.\left(\Psi q_{j}^{2} \Psi^{*} f_{k}\right)\right|_{-\infty} ^{\infty}-\int_{-\infty}^{\infty} \frac{\partial}{\partial q_{j}}\left(q_{j}^{2} f_{k}\right) \Psi^{*} \Psi d q_{1} \ldots d q_{n}= \\
= & -\int_{-\infty}^{\infty}\left\{\frac{\partial f_{k}}{\partial q_{j}} \Psi^{*} q_{j}^{2} \Psi+2 \Psi^{*} q_{j} f_{k} \Psi\right\} d q_{1} \ldots d q_{n}
\end{aligned}
$$

Подставив (15) в (14), с учетом (13) и (12) придем к уравнению (11).

ТЕОРема 5. Среднее значение $j$-й компоненты обобщенного импульса удовлетворяет уравнению

$$
d\left\langle p_{j}(t)\right\rangle / d t=\left\langle-\sum_{k=1}^{n}\left(p_{k} \partial f_{k} / \partial q_{j}\right)\right\rangle-\left\langle\partial U / \partial q_{j}\right\rangle
$$


ДокАЗАТЕльСтво. Найдем производную от среднего значения $j$-й компоненты случайного вектора обобщенного импульса и воспользуемся уравнениями (2) и (3). Имеем

$$
\begin{aligned}
d\left\langle p_{j}\right\rangle / d t= & -i \hbar_{q} \frac{d}{d t}\left(\int_{-\infty}^{\infty} \ldots \int_{-\infty}^{\infty} \Psi^{*}(\bar{q}, t) \partial \Psi(\bar{q}, t) / d q_{j} d q_{1} \ldots d q_{n}\right)= \\
= & -i \hbar_{q} \int_{-\infty}^{\infty} \cdots \int_{-\infty}^{\infty}\left[\frac{\partial \Psi^{*}}{\partial t} \frac{\partial \Psi}{\partial q_{j}}+\Psi^{*} \frac{\partial}{\partial q_{j}}\left(\frac{\partial \Psi}{\partial t}\right)\right] d q_{1} \ldots d q_{n}= \\
= & \int_{-\infty}^{\infty} \ldots \int_{-\infty}^{\infty}\left\{i \hbar_{q}\left(\sum_{k=1}^{n}\left(f_{k} \frac{\partial \Psi^{*}}{\partial q_{k}}+\frac{1}{2} \Psi^{*} \frac{\partial f_{k}}{\partial q_{k}}\right)\right)+\Psi^{*} U\right\} \frac{\partial \Psi}{\partial q_{j}} d q_{1} \ldots d q_{n}+ \\
& +\int_{-\infty}^{\infty} \ldots \int_{-\infty}^{\infty} \Psi^{*} \frac{\partial}{\partial q_{j}}\left(i \hbar_{q}\left(\sum_{k=1}^{n}\left(f_{k} \frac{\partial \Psi}{\partial q_{k}}+\frac{1}{2} \Psi \frac{\partial f_{k}}{\partial q_{k}}\right)\right)-\Psi U\right) d q_{1} \ldots d q_{n}= \\
= & i \hbar_{q} \sum_{k=1}^{n} \int_{-\infty}^{\infty} \ldots \int_{-\infty}^{\infty}\left\{f_{k}\left(\frac{\partial \Psi^{*}}{\partial q_{k}} \frac{\partial \Psi}{\partial q_{j}}-\frac{\partial \Psi}{\partial q_{k}} \frac{\partial \Psi^{*}}{\partial q_{j}}\right)+\right. \\
& \left.+\frac{1}{2} \frac{\partial f_{k}}{\partial q_{k}}\left(\Psi^{*} \frac{\partial \Psi}{\partial q_{j}}-\Psi \frac{\partial \Psi^{*}}{\partial q_{j}}-\right)\right\} d q_{1} \ldots d q_{n}+ \\
& +\int_{-\infty}^{\infty} \ldots \int_{-\infty}^{\infty}\left(\Psi^{*} U \frac{\partial \Psi}{\partial q_{j}}-\Psi^{*} \frac{\partial}{\partial q_{j}}(\Psi U)\right) d q_{1} \ldots d q_{n} .
\end{aligned}
$$

Легко показать, что

$$
\begin{aligned}
&\left\langle p_{j}\right\rangle=\left\langle-i \hbar_{q} \partial / \partial q_{j}\right\rangle=\frac{1}{2} \int_{-\infty}^{\infty} \ldots \int_{-\infty}^{\infty}\left\{-i \hbar_{q}\left(\Psi^{*} \frac{\partial \Psi}{\partial q_{j}}-\frac{\partial \Psi^{*}}{\partial q_{j}} \Psi\right)\right\} d q_{1} \ldots d q_{n} \\
& i \hbar_{q} \sum_{k=1}^{n} \int_{-\infty}^{\infty} \ldots \int_{-\infty}^{\infty}\left\{f_{k}\left(\frac{\partial \Psi^{*}}{\partial q_{k}} \frac{\partial \Psi}{\partial q_{j}}-\frac{\partial \Psi}{\partial q_{k}} \frac{\partial \Psi^{*}}{\partial q_{j}}\right)+\right. \\
& \quad+\frac{1}{2} \frac{\partial f_{k}}{\partial q_{k}}\left(\Psi^{*} \frac{\partial \Psi}{\partial q_{j}}-\Psi \frac{\partial \Psi^{*}}{\partial q_{j}}\right\} d q_{1} \ldots d q_{n}= \\
&=-\frac{1}{2} \sum_{k=1}^{n} \int_{-\infty}^{\infty} \cdots \int_{-\infty}^{\infty}\left(-i \hbar_{q}\left(\Psi^{*} \frac{\partial f_{k}}{\partial q_{j}} \frac{\partial \Psi}{\partial q_{k}}-\frac{\partial f_{k}}{\partial q_{j}} \frac{\partial \Psi^{*}}{\partial q_{k}} \Psi\right)\right) d q_{1} \ldots d q_{n}= \\
&=-\left\langle\sum_{k=1}^{n} p_{k} \frac{\partial f_{k}}{\partial q_{j}}\right\rangle^{\infty}, \int_{-\infty}^{\infty}\left(\Psi^{*} \frac{\partial \Psi}{\partial q_{j}}-\Psi^{*} \frac{\partial}{\partial q_{j}}(\Psi U)\right) d q_{1} \ldots d q_{n}= \\
& \int_{-\infty}^{\infty} \ldots \int_{-\infty}^{\infty} \Psi^{*} \frac{\partial U}{\partial q_{j}} \Psi d q_{1} \ldots d q_{n} .
\end{aligned}
$$

В итоге получаем

$$
\frac{d\left\langle p_{j}(t)\right\rangle}{d t}=-\left\langle\sum_{k=1}^{n} p_{k} \frac{\partial f_{k}}{\partial q_{j}}\right\rangle-\left\langle\frac{\partial U}{\partial q_{j}}\right\rangle .
$$




\section{3. СООТНОШЕНИЕ НЕОПРЕДЕЛЕННОСТЕЙ И СПОСОБ ЗАДАНИЯ $\hbar_{q}$}

Введем обозначения для дисперсий взаимно сопряженных компонент обобщенной координаты $q_{j}$ и обобшенного импульса $p_{j}$

$$
\begin{aligned}
\sigma_{q_{j}}^{2}= & \int_{-\infty}^{\infty} \cdots \int_{-\infty}^{\infty} \Psi^{*}\left(q_{j}-\int_{-\infty}^{\infty} \ldots \int_{-\infty}^{\infty} \Psi^{*} q_{j} \Psi d q_{1} \ldots d q_{n}\right)^{2} \Psi d q_{1} \ldots d q_{n} \\
\sigma_{p_{j}}^{2}= & \int_{-\infty}^{\infty} \ldots \int_{-\infty}^{\infty} \Psi^{*}\left(-i \hbar_{q}\left(\frac{\partial}{\partial q_{j}}\right)-\right. \\
& \left.-\int_{-\infty}^{\infty} \cdots \int_{-\infty}^{\infty} \Psi^{*}\left(-i \hbar_{q}\left(\frac{\partial}{\partial q_{j}}\right)\right) \Psi d q_{1} \ldots d q_{n}\right)^{2} \Psi d q_{1} \ldots d q_{n} .
\end{aligned}
$$

Повторяя выкладки, приведенные в [9], докажем следующую теорему.

ТЕОрема 6. Произведение дисперсий взаимно сопряженных компонент обобщенных координать и импульса не меньше величинь $\hbar_{q}$, т.е.

$$
\sigma_{q_{j}}^{2} \cdot \sigma_{p_{j}}^{2} \geq \hbar_{q}^{2} / 4
$$

Из теоремы 6 можно получить важное соотношение, позволяюшее задать $\hbar_{q}$, если известна начальная дисперсия $\sigma_{q_{j}}^{2}(t=0)$.

ОПРЕДЕЛЕНИЕ 5. Пусть известны начальные значения дисперсий взаимно сопряженных компонент обобшенных координат и импульса $\sigma_{q_{j}}^{2}(t=0)=\sigma_{q_{j 0}}^{2} \neq 0$ и $\sigma_{p_{j}}^{2}(t=$ $0)=\sigma_{p_{j 0}}^{2} \neq 0$. Определим $\hbar_{q}$ следуюшим образом:

$$
\hbar_{q}=2 \min _{(j=1,2, \ldots, n)}\left(\sigma_{q_{j 0}} \cdot \sigma_{p_{j 0}}\right) .
$$

В частности, если положить $\sigma_{p_{j 0}}=1$, получим

$$
\hbar_{q}=2 \min _{(j=1,2, \ldots, n)} \sigma_{q_{j 0}} .
$$

Рассмотрим линеаризованную систему (1)

$$
d\left\langle q_{j}(t)\right\rangle / d t=\left\langle\sum_{k=1}^{n} \partial f_{j}\left(\left\langle q_{1}(t)\right\rangle, \ldots,\left\langle q_{n}(t)\right\rangle, t\right) / \partial q_{k}\left(q_{k}-\left\langle q_{k}(t)\right\rangle\right)\right\rangle, \quad j=1,2, \ldots, n .
$$

Введем обозначения $a_{j i}=\partial f_{j}\left(\left\langle q_{1}(t)\right\rangle, \ldots,\left\langle q_{n}(t)\right\rangle, t\right) / \partial q_{i}$. Будем считать, что все собственные векторы матрищы $\left(a_{j i}\right)$ линейно независимы. Тогда с помошью замены переменных, которая является переходом к базису собственных векторов, матрица $\left(a_{j i}\right)$ может быть приведена к диагональному виду. Без ограничения общности вместо линеаризованной системы (1) рассмотрим систему вида

$$
d\left\langle q_{j}(t)\right\rangle / d t=\lambda_{j}\left\langle q_{j}\right\rangle,
$$

где $\lambda_{j}$ - собственные значения матрицы $\left(a_{j i}\right)$.

Следующая теорема дает условие устойчивости волновых пакетов в линейном приближении. 
ТЕОРЕМА 7. Если выполняются условия

$$
-\infty<\lambda_{1 j} \leq \lambda_{j} \leq \lambda_{2 j}<0
$$

mo

$$
\lim _{\substack{t \rightarrow \infty \\ \hbar_{q} \rightarrow 0}} \sigma_{q_{j}}^{2}(t)=0 .
$$

Если жсе

$$
0<\lambda_{3 j} \leq \lambda_{j} \leq \lambda_{4 j}<\infty
$$

mo

$$
\lim _{\substack{t \rightarrow \infty \\ \hbar_{q} \rightarrow 0}} \sigma_{q_{j}}^{2}(t)=\infty
$$

ДокАЗАТЕЛЬСтво. Докажем соотношение (18). Имеем

$$
\begin{aligned}
d \sigma_{q_{j}}^{2} / d t= & 2\left(\left\langle f_{j}(\bar{q}, t) q_{j}\right\rangle-\left\langle q_{j}\right\rangle\left\langle f_{j}(\bar{q}, t)\right\rangle\right)= \\
= & 2 \int_{-\infty}^{\infty} \cdots \int_{-\infty}^{\infty} \Psi^{*} q_{j} \lambda_{j} q_{j} \Psi d q_{1} \ldots d q_{n}- \\
& -2 \int_{-\infty}^{\infty} \cdots \int_{-\infty}^{\infty} \Psi^{*} q_{j} \Psi d q_{1} \ldots d q_{n} \times \\
& \times \int_{-\infty}^{\infty} \cdots \int_{-\infty}^{\infty} \Psi^{*} \lambda_{j} q_{j} \Psi d q_{1} \ldots d q_{n}=2 \lambda_{j} \sigma_{q_{j}}^{2} .
\end{aligned}
$$

Из соотношений (16), (17) и (20) следует

$$
\sigma_{q_{j}}^{2} \leq \sigma_{q_{j}}^{2}(0) \exp \left(-2\left|\lambda_{2 j}\right| t\right)=\left(\hbar_{q}^{2} / 4\right) \exp \left(-\left|\lambda_{2 j}\right| t\right) \rightarrow 0
$$

при $t \rightarrow \infty$ и $\hbar_{q} \rightarrow 0$.

Аналогично доказывается соотношение (19).

СЛЕДСТВИЕ. Если выполняются условия

$$
-\infty<\lambda_{1 j} \leq \partial f_{j} / \partial q_{k} \leq \lambda_{2 j}<0, \quad q_{j} \in(-\infty, \infty), \quad k=1,2, \ldots, n, \quad j=1,2, \ldots, n,
$$

mo

$$
\lim _{\substack{t \rightarrow \infty \\ \hbar_{q} \rightarrow 0}} \sigma_{q_{j}}^{2}(t)=0
$$

Если же справедливь неравенства

$$
0<\lambda_{3 j} \leq \partial f_{j} / \partial q_{k} \leq \lambda_{4 j}<\infty, \quad q_{j} \in(-\infty, \infty), \quad k=1,2, \ldots, n, \quad j=1,2, \ldots, n,
$$

mo

$$
\lim _{\substack{t \rightarrow \infty \\ \hbar_{q} \rightarrow 0}} \sigma_{q_{j}}^{2}(t)=\infty
$$


Рассмотрим один важный пример, относяшийся к описанию случайного одномерного процесса со стационарным гауссовским распределением

$$
P(q, t)=\frac{1}{2} \hbar_{q}(2 \pi)^{1 / 2} \exp \left(-2(q-a)^{2} / \hbar_{q}^{2}\right)
$$

Если положить $P(q, t)=\Psi(q, t) \Psi^{*}(q, t)$, а затем подставить $\Psi^{*}(q, t)=P(q, t) / \Psi(q, t)$ в уравнение (3), то получим с учетом (2) следуюшее обыкновенное дифференциальное уравнение для функции $f(q): f(q)(q-a) / \sigma^{2}-d f / d q=0$. Заметим, что из общего решения этого уравнения $f(q)=C \exp \left((q-a)^{2} /\left(2 \sigma^{2}\right)\right)$ условию существования среднего значения $\langle f(q)\rangle$ удовлетворяет лишь $f(q) \equiv 0$. Подставив $f(q)=0$ в уравнение (2), получим следующее уравнение для временного множителя $T(t)$ волновой функции $\Psi(q, t)=T(t) \sqrt{P(q, t)}: i \hbar_{q} d T / d t-U T=0$, отсюда $T(t)=\operatorname{const} \exp \left(-i U(t) / \hbar_{q}\right)$ и с точностью до нормировочного множителя

$$
\Psi(q, t)=\exp \left(-i U(t) / \hbar_{q}\right)\left[\frac{1}{2} \hbar_{q}(2 \pi)^{1 / 2} \exp \left(-2(q-a)^{2} / \hbar_{q}^{2}\right)\right]^{1 / 2}
$$

Найденная функция описывает колебания с частотой $\omega-U / \hbar_{q}$ около среднего значения $\langle q\rangle=a$. Соответственно для полного описания поведения случайной величины $q$ даже в случае стационарности $\langle q(t)\rangle=a=$ const наблюдаемого во времени среднего значения необходимо задание не только начальной дисперсии, но и частотной характеристики величины $q$, определяемой величиной $U(q)$. Таким образом, $U(q)$ задает частоту флуктуаций около среднего стационарно наблюдаемого значения случайной величины.

Подобная ситуация может иметь место в эксперименте с фиксированной в электростатическом поле броуновской частицей в середине $(q=a)$ узкого сосуда с жидкостью, находящейся при постоянной температуре. Вверху и внизу сосуда расположены два одноименных с зарядом частицы более мошных заряда. Толшина сосуда чуть больше диаметра частицы. Постоянные толчки со стороны молекул приводят к незначительным отклонениям частицы от положения равновесия, в которое она постоянно приводится фиксирующим электростатическим полем (или с помощью других подобных упругим силам воздействий). Среднее значение, дисперсию и частоту колебаний можно экспериментально определить путем измерений. Заданием этих величин наблюдаемый случайньй процесс тем самым будет полностью определен, и его описание предпочтительнее вести с помошью предложенного здесь подхода.

\section{Список литературы}

[1] А. Эйнштейн, М. Смолуховский. Броуновское движение. М.-Л.: ОНТИ, 1936.

[2] P. Langevin. C. R. Acad. Sci. 1908. V. 146. P. 530.

[3] Г. Хакен. Синергетика. Иерархии неустойчивостей в самоорганизующихся системах и устройствах. М.: Мир, 1985.

[4] A. D. Fokker. Ann. Phys. (Leipzig). 1915. V. 43. P. 310.

[5] М. Планк. Термодинамика. Теория излучения и квантовая теория. Теория относительности Избр. труды. М.: Наука, 1975.

[6] A. N. Kolmogorov. Math. Annal. 1931. V. 104. P. 415. 
[7] К. Ито. Вероятностные процессы. Вып. 1. М.: ИЛ, 1960.

[8] Р. Л. Стратонович. Избранные вопросы теории флуктуаций в радиотехнике. М.: Сов. радио, 1961.

[9] Л. де Бройль. Соотношение неопределенностей Гейзенберга и вероятностная интерпретация волновой механики. М.: Мир, 1986.

[10] П. Эренфест. Относительность. Кванты. Статистика. М.: Наука, 1972.

[11] Г. Николис, И. Пригожин. Самоорганизация в неравновесных системах. М.: Мир, 1979.

[12] К. В. Гардинер. Стохастические методы в естественных науках. М.: Мир, 1986.

[13] G. Nicolis, I. Prigogine. Exploring Complexity. An introduction. New York: W. H. Freeman and Company, 1989.

[14] И. А. Соловьев. Вестн. МЭИ. 1995. № 6. С. 109-118.

[15] И. А. Соловьев. Сб. научн. тр. МГОПИ. 1996. № 12. С. 81-92.

Поступила в редакцию 17.I.1997 г.

\section{A. Soloviev}

\section{STOCHASTIC WAVE EQUATION SIMULATING THE BEHAVIOR OF QUANTITIES WITH THE AVERAGES OBEYING THE SET OF ORDINARY FIRST ORDER DIFFERENTIAL EQUATIONS}

The theory of stochastic waves for stochastic vector quantities satisfying the set of ordinary first order differential equations is presented. The equation for wave functions corresponding to a differential model for mean values is suggested. The relationship between this equation and Liouville's equation is considered. The analog of Ehrenfest's theorem is proved. The ordinary first order differential equation for dispersion is obtained. The problems of interpretation and determination of the analog of Planck's constant are disscussed. The conditions for increasing and decreasing of dispersion are found. 